\title{
Grupo de mulheres na comunidade: (re)construindo saberes em saúde
}

\section{Group of women in the community: (re)building knowledge in health}

\author{
Joyce Mazza Nunes ${ }^{1}$, Eliany Nazaré Oliveira ${ }^{2}$, Neiva Francenely Cunha Vieira ${ }^{3}$
}

\begin{abstract}
Resumo
Objetiva-se descrever a experiência vivenciada com um grupo de mulheres de uma comunidade de Fortaleza (CE) na realização de atividades educativas em saúde, com o intuito de fortalecer o autocuidado e a promoção da saúde das muIheres, além de contribuir para o aumento da autonomia e cidadania. Os temas discutidos foram eleitos pelas participantes, referente às temáticas câncer de mama e infertilidade. O aprendizado foi elaborado mediante o diálogo e compartilhamento de experiências entre os sujeitos e os profissionais de saúde, subsidiado por jogos educativos. Espera-se contribuir para o desenvolvimento da prática educativa em saúde com a comunidade, demonstrando que isso é possível, valorizando a participação dos sujeitos, sua realidade e necessidades.
\end{abstract}

Palavras-chave: educação em saúde; promoção da saúde; saúde da mulher.

\begin{abstract}
The objective is to report the lived experience with women of a community of Fortaleza in educational activities in health, with the aim of strengthening self-care and health promotion of women, and contribute to the increased autonomy and citizenship. The topics discussed were elected by the participants, concerning breast cancer and infertility. The learning was developed through dialogue and sharing of experiences among individuals and health professionals, supported by educational games. It is expected to contribute to the development of educational practice in health in the community, demonstrating that it is possible, valuing the participation of the subjects, their reality and needs.
\end{abstract}

Keywords: health education; health promotion; women's health.

Trabalho realizado no Programa de Pós-graduação em Enfermagem da Universidade Federal do Ceará (UFC) - Fortaleza (CE), Brasil.

'Doutoranda do Programa de Pós-graduação em Enfermagem da UFC; Enfermeira da Estratégia Saúde da Família de Fortaleza - Fortaleza (CE), Brasil.

2Doutora em Enfermagem pela UFC - Fortaleza (CE), Brasil; Professora do curso de Enfermagem da Universidade Estadual Vale do Acaraú - Sobral (CE), Brasil.

${ }_{3}^{3 P h D}$ pela Universidade de Bristol - Bristol, Inglaterra; Professora do curso de Enfermagem da UFC - Fortaleza (CE), Brasil.

Endereço para correspondência: Joyce Mazza Nunes - Rua Chico Lemos, 1405, casa 6 - Cidade dos Funcionários - CEP: $60822-780$ - Fortaleza (CE), Brasil. -

E-mail: joycemazza@hotmail.com

Conflito de interesses: nada a declarar. 


\section{INTRODUÇÃO}

O trabalho em saúde deve estimular a capacidade de reflexão e ação autônoma dos sujeitos envolvidos, trabalhadores e usuários, pois estes são sempre responsáveis por sua formação e pela constituição do mundo que os cerca ${ }^{1}$. Nessa perspectiva, estão as ações de educação em saúde como uma das intervenções potencialmente decisivas na promoção da saúde, pois se fazem a partir da análise, problematização e proposição dos profissionais de saúde e comunidade, sujeitos do processo ${ }^{2}$.

A Educação em Saúde, como prática na qual existe a participação ativa da comunidade, proporciona informação em saúde, educação sanitária e, principalmente, contribui para a aquisição de atitudes indispensáveis para a vida das pessoas ${ }^{3}$. Dessa forma, o desenvolvimento de práticas educativas em saúde, como instrumento de participação popular, e, ao mesmo tempo, favorecendo uma troca entre o saber científico e popular, deve ser estimulado na Estratégia Saúde da Família (ESF), com vistas à melhoria da qualidade de vida da comunidade, das famílias e dos indivíduos ${ }^{4}$.

A vista do exposto, embora se reconheça a importância do desenvolvimento de atividades educativas em saúde, especialmente na ESF, muitas das práticas desenvolvidas por enfermeiros ainda não incorporam a compreensão dos determinantes em saúde ou as necessidades e saberes dos sujeitos ${ }^{5}$.

Diante dessa realidade, é preciso despertar nos profissionais de saúde o interesse pela educação em saúde mais efetiva e resolutiva, por meio da integração dessas ações com a comunidade que possam contribuir para a manutenção da saúde individual e coletiva com consciência crítica, permitindo o exercício da cidadania e efetivando mudanças pessoais e sociais ${ }^{6,7}$.

As ações em saúde devem ser direcionadas para a realidade cultural dos sujeitos, na qual o problema é trabalhado a partir do pensamento do coletivo, da análise de suas crenças e valores sobre determinada situação ou risco de agravo à saúde e de práticas em relação à situação vivenciada ${ }^{8}$.

Os recursos tecnológicos utilizados nas atividades de Educação em Saúde devem favorecer a participação dos sujeitos no processo educativo, contribuindo para a conquista da cidadania e para o aumento da autonomia dos envolvidos, explorando recursos que vão ao encontro dos significados culturais.

As mulheres são a maioria da população brasileira e as principais usuárias do Sistema Único de Saúde (SUS). Frequentam os serviços de saúde para o seu próprio atendimento, mas, sobretudo, acompanhando maridos, filhos e pessoas idosas e/ou com deficiências em consultas. São também cuidadoras, não só das crianças ou outros membros da família, mas também de pessoas da vizinhança e da comunidade?
Elas merecem atenção especial, principalmente por suas necessidades de assistência em saúde, como contracepção, ciclo gravídico-puerperal, prevenção do câncer de mama e colo do útero, entre outras.

O Ministério da Saúde considera a saúde da mulher como prioridade e nos anos 80 criou o Programa de Atenção Integral à Saúde da Mulher (PAISM), sendo um marco histórico das políticas de gênero no país. Tal programa inseriu uma nova abordagem à saúde da mulher, que incorpora num enfoque de gênero a integralidade e a promoção da saúde como princípios norteadores e busca consolidar os avanços no campo dos direitos sexuais e reprodutivos 9 .

Com base nesses pressupostos, desenvolveram-se atividades educativas em saúde com um grupo de mulheres residentes em uma comunidade assistida pela ESF, com o intuito de contribuir para a promoção da saúde desse grupo de usuárias. Objetiva-se, neste texto, relatar essa experiência vivenciada durante dois encontros com o grupo de mulheres, a partir de um recorte de um estudo mais amplo.

\section{MÉTODO}

Trata-se de um estudo do tipo descritivo com abordagem qualitativa, em que se desenvolveram atividades educativas promovidas por uma equipe da ESF da periferia de Fortaleza (CE) (uma enfermeira e três mulheres que atuam como Agentes Comunitários de Saúde - ACS), com 11 mulheres adultas jovens da comunidade, com idades de 20 a 38 anos, durante o segundo semestre de 2009.

Os sujeitos foram convidados pelos ACS a participarem de um grupo de mulheres para o desenvolvimento de atividades educativas em saúde, a partir de temas de interesse das participantes referentes à saúde da mulher.

As atividades educativas aconteceram na própria comunidade, no turno da tarde, com duração de 3 horas. A opção foi pelo período da tarde, pois, nessa parte do dia, já cumpriram com suas "tarefas do lar", estando disponíveis para participar das atividades. Criou-se um ambiente alegre e descontraído, favorecendo a participação e a aprendizagem. Buscou-se fortalecer o autocuidado e a promoção da saúde das mulheres, contribuindo para o aumento da autonomia e cidadania.

Essas atividades foram subsidiadas por recursos tecnológicos, compreendidos como um conjunto de conhecimentos que se aplicam a uma atividade educativa partindo da realidade do ser humano, valorizando sua experiência, seu contexto de vida e suas expectativas frente ao processo saúde-doença ${ }^{10}$. Constituiu-se de dinâmicas, jogos, exposição de filmes, utilização da literatura de cordel, dramatização, entre outros. A escolha desses 
recursos deu-se baseada na realidade das mulheres e no tema selecionado para discussão.

O saber de cada mulher foi reconhecido e valorizado e, com arrimo nessa realidade e mediante o diálogo, procurou-se transformar e/ou complementar esse saber, estabelecendo a troca de conhecimento popular e científico.

Somente o primeiro encontro foi planejado exclusivamente pela equipe da ESF, sendo os demais em parceria com todas as participantes, de forma bem particularizada, de acordo com as temáticas sugeridas pelo grupo, levando-se em conta a singularidade, a realidade e as necessidades de cada mulher e as informações obtidas em encontros anteriores.

Para coleta de informações, utilizaram-se registros dos encontros do grupo (roteiro de identificação, gravação dos diálogos) e o diário de campo da pesquisadora. Foram selecionados os aspectos relevantes e apresentados no estudo. A coleta e a análise das informações ocorreram de forma simultânea, no decorrer dos encontros no grupo de mulheres, pois, assim, poderia modificar as estratégias utilizadas e possíveis entraves que pudessem ocorrer na condução do grupo, bem como refletir, fazer interpretações e identificar lacunas que poderiam ser preenchidas a tempo.

O estudo foi norteado pela Resolução $n^{\circ}$ 196/96, do Conselho Nacional de Saúde, sobre pesquisa envolvendo seres humanos ${ }^{11}$. O projeto foi aprovado pelo Comitê de Ética em Pesquisa da Universidade Federal do Ceará (UFC), mediante protocolo $n^{\circ} 153 / 09$. As mulheres assinaram o termo de consentimento livre e esclarecido. Para preservar o anonimato das participantes do estudo, elas foram identificadas pela letra $\mathrm{M}$ numeradas sequencialmente, ou seja, M1, M2, M3 e assim por diante até M11.

Neste estudo, apresenta-se a experiência de dois encontros com o grupo de mulheres, cujos temas abordados foram câncer de mama e infertilidade, ambos sugeridos pelas participantes por serem relevantes para a comunidade.

\section{RESULTADOS}

\section{Vivência de atividades educativas com um grupo de mulheres}

Para discutir a temática câncer de mama, eleita pelas participantes por ser relevante para a comunidade, elas sugeriram que fosse desenvolvido um jogo. Dessa forma, a equipe da ESF desenvolveu previamente um jogo contendo perguntas sobre o tema, de modo a formular novos conhecimentos e valorizar a participação, o saber e a experiência de cada mulher e esclarecer possíveis dúvidas.

O jogo é uma adaptação do conhecido jogo de tabuleiro que se desenvolve à medida que a participante responde a uma pergunta e, assim, avança uma casa do tabuleiro. Vence a participante que conseguir chegar primeiro à última casa $\mathrm{da}$ tábua e responder à última pergunta.

O grupo elegeu duas participantes para disputar o jogo, que previa, também, a utilização de uma prótese de silicone e de pano, na prancha, disponibilizado pelo Centro de Saúde da Família (CSF) local, para auxiliar na aprendizagem da realização do exame das mamas e identificação de nódulos mamários. As demais mulheres e os profissionais de saúde apoiavam as competidoras, complementando esse conhecimento e esclarecendo dúvidas.

Tudo transcorreu de maneira divertida, agradável e descontraída, à medida que as mulheres iam respondendo as perguntas, aumentava a sua participação. As participantes relataram seus saberes e práticas, suas experiências sobre 0 tema, citando casos de mulheres conhecidas no bairro que tiveram câncer de mama.

Foi distribuído entre as participantes um informativo sobre como realizar corretamente o autoexame das mamas, além de ter ocorrido a demonstração, pelos profissionais de saúde, da técnica correta do autoexame das mamas.

No fim do jogo, solicitou-se que todas as mulheres relatassem: 1) o que já sabiam sobre câncer de mama anteriormente, 2) o que aprenderam no encontro e 3) o que pretendiam adotar como práticas de prevenção do câncer de mama. Desse modo, identificou-se o aprendizado resultado do encontro com as mulheres, apresentado no Quadro 1.

Percebe-se que as mulheres pouco conheciam sobre o câncer de mama. Elas sabiam que se tratava de uma enfermidade que causava a morte de muitas mulheres, mas pouco sabiam sobre o autoexame das mamas (AEM). O jogo permitiu a ampliação desses conhecimentos. As mulheres também relataram suas intenções para mudanças de atitudes que favoreçam a prevenção do câncer de mama, embasadas no conhecimento constituído no encontro, mediante o desenvolvimento do jogo.

No fim, as duas participantes competidoras foram indagadas sobre como foi para elas participar daquela atividade e elas responderam o seguinte: "Jogo é legal, é aprender brincando" (M7) e "A gente aprende e se diverte ao mesmo tempo" (M2). Outro tema sugerido pelas participantes para ser discutido no grupo, que também representaria um problema na saúde da mulher para aquela comunidade, foi a infertilidade, pois relataram conhecer casais inférteis na sua comunidade e gostariam de obter maiores informações sobre esse problema de saúde, para poderem ajudar, de alguma forma, essas pessoas conhecidas.

Para contextualizar esse tema, realizou-se o conhecido "jogo do repolho", adaptado pela equipe da ESF para a temática da infertilidade. À medida que eram feitas as perguntas no jogo, as mulheres respondiam com o seu conhecimento e 
Quadro 1. Elaboração compartilhada de conhecimento sobre câncer de mama e adoção de práticas preventivas - Fortaleza (CE), 2009

\begin{tabular}{|c|c|c|c|}
\hline Mulheres & O que eu já sabia & O que eu aprendi & O que eu adoto como prática preventiva \\
\hline M2 & $\begin{array}{l}\text { Sobre o AEM, mas não fazia certo, } \\
\text { tinha dúvidas. }\end{array}$ & $\begin{array}{l}\text { Como fazer corretamente o AEM e como } \\
\text { identificar os nódulos. }\end{array}$ & $\begin{array}{l}\text { Ter uma alimentação saudável, fazer exercício } \\
\text { físico e me cuidar mais e me prevenir. }\end{array}$ \\
\hline M3 & $\begin{array}{l}\text { Que amamentar diminuía o risco } \\
\text { de ter câncer de mama. }\end{array}$ & $\begin{array}{l}\text { Sobre a importância de hábitos e } \\
\text { alimentação saudáveis. }\end{array}$ & $\begin{array}{l}\text { Diminuir o uso de bebidas alcoólicas e fazer } \\
\text { o AEM mensalmente. }\end{array}$ \\
\hline M4 & $\begin{array}{l}\text { Que era um câncer que matava } \\
\text { muitas mulheres. }\end{array}$ & $\begin{array}{l}\text { Sobre os fatores de risco para câncer de } \\
\text { mama e a importância do exercício físico } \\
\text { e da alimentação saudável. }\end{array}$ & $\begin{array}{l}\text { Mudança de hábitos alimentares, } \\
\text { valorizando a alimentação rica em frutas e } \\
\text { verduras, praticar exercícios físicos. }\end{array}$ \\
\hline M5 & $\begin{array}{l}\text { Que era o câncer que mata muitas } \\
\text { mulheres e que tinha que fazer o } \\
\text { AEM, mas nunca fazia. }\end{array}$ & $\begin{array}{c}\text { Como fazer o AEM e mamografia a } \\
\text { partir do } 40 \text { anos, mudança de hábitos } \\
\text { alimentares. }\end{array}$ & $\begin{array}{l}\text { Fazer o AEM mensalmente, tentar mudar os } \\
\text { hábitos alimentares. É difícil, mas eu vou tentar. }\end{array}$ \\
\hline M7 & $\begin{array}{l}\text { Que a mulher que amamenta tem } \\
\text { menos riscos de ter câncer de mama, } \\
\text { sobre o AEM, mas pouco fazia. }\end{array}$ & $\begin{array}{l}\text { Sobre os fatores de riscos para câncer de } \\
\text { mama, os sintomas do câncer de mama e } \\
\text { a maneira correta de fazer o AEM. }\end{array}$ & $\begin{array}{l}\text { Mudança de hábitos alimentares e } \\
\text { diminuição do uso de bebidas alcoólicas. }\end{array}$ \\
\hline M8 & Um pouco sobre o AEM. & $\begin{array}{l}\text { A frequência certa da realização do } \\
\text { AEM e a identificar os nódulos com a } \\
\text { ajuda da prótese de silicone. }\end{array}$ & $\begin{array}{l}\text { Fazer frequentemente o AEM, ter uma } \\
\text { alimentação saudável e fazer caminhada. }\end{array}$ \\
\hline M11 & Um pouco sobre o AEM. & $\begin{array}{l}\text { Sobre o AEM, a importância de } \\
\text { alimentação e hábitos saudáveis. }\end{array}$ & $\begin{array}{l}\text { Fazer o AEM frequentemente e melhorar os } \\
\text { hábitos alimentares. }\end{array}$ \\
\hline
\end{tabular}

AEM: autoexame das mamas

os profissionais de saúde complementavam, o que permitiu a elaboração conjunta do saber.

No decorrer da discussão, surgiram dúvidas referentes aos órgãos genitais, reprodução humana e métodos anticoncepcionais, esclarecidas pelos profissionais de saúde presentes, utilizando linguagem clara, evitando termos técnicos.

A penúltima pergunta do jogo era: por que é importante discutir a infertilidade no grupo de mulheres? As mulheres acreditam na relevância de produzir essa discussão no grupo para poderem compartilhar experiências e, assim, contribuir com outras pessoas da comunidade. "A gente aprende e passa para outras pessoas que não sabem e a gente pode informar o que a gente aprendeu aqui" (M2); "Daqui a pouco a gente tem um diploma, igual a elas" (aponta para as acadêmicas de enfermagem, que estavam presentes no encontro) (M3); “O pessoal do bairro já vai saber que a gente sabe e ao invés de ir pro posto de saúde, vão se consultar lá em casa” (M1).

É importante destacar o comentário de uma das mulheres, que se referiu à necessidade de debater sobre infertilidade no grupo. "Eu que sugeri o tema, porque todo encontro que a gente vai, a gente só discute planejamento familiar, DST etc. e infertilidade é a primeira vez que eu vejo alguém discutir" (M6).

Elas também perceberam a crescente participação nos diálogos e a evolução pessoal das próprias participantes.

A fulana quando entrou aqui não abria nem a boca, nem pegava no papel, e agora ela está falando, e agora é a outra, que mal está falando, mas daqui a uns dois encontros ela estará falando também (M5).
A última pergunta do jogo era: como o grupo pode alertar as pessoas sobre a infertilidade? Essa pergunta tentava fomentar uma reflexão sobre a possibilidade de protagonismo das mulheres participantes do grupo. Muitas fizeram comentários. "Às vezes, a gente sentada na calçada com as amigas, fala do grupo, mesmo sem elas estarem precisando, então elas já vão aprendendo e vão repassando pras outras” (M6). “Tem um ditado que diz que o boca a boca tem poder, uma fala pra uma, outra fala pra outra e assim vai” (M7).

As mulheres não ficaram tímidas para falar sobre sexualidade, que envolve a temática infertilidade. $O$ tema infertilidade foi discutido mediante um jogo de perguntas e respostas, o que possibilitou criar um ambiente descontraído. A participante que mais se envolveu foi aquela que sugeriu o tema infertilidade.

Em seguida, a equipe da ESF propôs-se a realização de uma dramatização sobre infertilidade, para que as mulheres demonstrassem o seu aprendizado constituído no jogo. Seis voluntárias encenaram um caso fictício de um casal infértil. Posteriormente, refletiram sobre a encenação e acerca do caso de infertilidade, enfatizando a maneira como cada uma se comportou diante de um caso de infertilidade na família.

Também foi distribuído entre as participantes um texto de cordel sobre infertilidade, composto pela pesquisadora. As mulheres aceitaram bem o cordel, que continha versos sobre infertilidade. Elas conheciam a literatura de cordel, que fazia parte do seu contexto, e isso facilitou a compreensão da temática exposta.

No fim do encontro, algumas participantes surpreenderam a todos com sua manifestação espontânea sobre o encontro, iniciando novo diálogo: 
Eu queria dizer que eu gostei muito... fui eu que sugeri esse tema, eu sabia que eu iria aprender, mas não sabia como, mas cada comentário de cada um acrescenta bastante no aprendizado da gente (M10);

É difícil uma mãe de família, como muitas aqui são, ter um tempo pra sentar, pra brincar e pra sorrir, pra conversar com alguém, é mais é problema de casa (M4);

Eu acho que os temas que já foram discutidos aqui de alguma forma contribuíram na vida de cada uma e também na troca de informações, pois sempre a gente conhece alguém que passa por aquela situação e a gente fala e ajuda (M9).

\section{DISCUSSÃO}

Mediante os comentários, percebeu-se que as mulheres aprovaram a maneira como os encontros foram desenvolvidos e sentem-se motivadas para participar, pois estão inclusas nesse processo. Os jogos facilitaram a aprendizagem, incentivando a participação dos sujeitos, tornando o encontro descontraído e alegre, representando um momento lúdico no dia a dia das mulheres, que é muito agitado. Esse componente lúdico também favorece o acolhimento dos sujeitos e de suas experiências, tornando-se possível compartilhar saberes ${ }^{12}$.

Outro ponto que merece ser destacado é a dificuldade que elas enfrentam para conciliar o cuidado do lar e da própria saúde, pois são socialmente designadas como cuidadoras da família. Relatam que, no dia a dia de dona de casa, não há tempo para dialogar com amigas, pois há muitos afazeres do lar para serem realizados. Nos encontros do grupo, elas puderam dar uma pausa nessas tarefas e aprenderam mais sobre sua saúde de uma maneira divertida, conquistando novas amizades.

Desse modo, os encontros representaram um momento terapêutico e de lazer para as mulheres, que diariamente têm uma rotina cansativa e estressante, no cuidado com o seu lar e com a família. No grupo, elas poderiam dar uma pausa nessas atribuições e encontrar outras mulheres para dialogar e se divertir. $O$ fato de participar de um grupo com outras mulheres do bairro, por si, já contribui para a promoção da saúde das mulheres, pois o grupo promove, além de tudo, a integração e socialização das pessoas.

A participação no grupo contribui para a promoção da saúde mental das mulheres, na medida em que as participantes tem a oportunidade de vivenciar momentos de contentamento e alegria ${ }^{13}$.

No decorrer dos encontros, as mulheres puderam ir se conhecendo e estabelecendo vínculos de amizade e confiança, o que contribuiu para que elas, cada vez mais, participassem das discussões. Espaços de educação em saúde também são entendidos enquanto local de interação e trocas de experiências ${ }^{14}$.

As discussões valorizaram o conhecimento prévio dos sujeitos, identificando seus saberes, para, então, oferecer subsídios para a complementação desses saberes e/ou transformar saberes preexistentes. Para que as pessoas possam fazer escolhas mais saudáveis de vida, é necessário que haja um processo de interação entre o conteúdo teórico e a experiência de vida de cada um e o estabelecimento da confiança e da vinculação do usuário ao serviço de saúde e ao profissional ${ }^{15}$.

Nos encontros, buscou-se identificar os interesses e necessidades das participantes, que devem ser valorizados. Os temas das atividades educativas devem partir da própria realidade dos envolvidos e não devem ser previamente estabelecidos pelos profissionais, a demanda deve ser colocada de forma espontânea e a partir das necessidades do grupo ${ }^{16}$.

As informações sobre saúde necessitam ser trabalhadas de forma simples e contextualizadas, instrumentalizando as pessoas para fazerem escolhas mais saudáveis de vida ${ }^{15}-$ foi assim que se procurou nortear as discussões. No grupo de mulheres, foram discutidos temas em saúde da mulher, sugeridos por elas próprias e subsidiados por recursos educativos (jogos, literatura de cordel etc) embasados culturalmente, de acordo com seu contexto, realidade e interesse. Desse modo, elas participavam e envolviam-se mais ativamente, pois se sentiam também responsáveis pelo desenvolvimento daquele encontro.

O desafio para a ação educativa em saúde é promover a aproximação entre o saber do senso comum e o saber profissional, que permita a "interpenetração" de saberes, possibilitando aos sujeitos adotar o diálogo como estratégia para transformação da realidade em saúde ${ }^{17}$.

Elas reconhecem que estão aprendendo umas com as outras, em grupo, e que estão cada vez mais aumentando sua participação nos encontros e exercitando sua autonomia, acreditando ser referência na comunidade. Assim, enfatiza-se que a perspectiva normativa e autoritária, que visa à transmissão de informações, em nada favorece o aprendizado ${ }^{14}$.

Participar e conduzir o grupo de mulheres fez perceber que não é apenas importante discutir os temas propostos no grupo sobre saúde da mulher, é preciso saber ouvir os sujeitos, estar disponível para esclarecer suas dúvidas e atentar para a dinâmica das relações no grupo e suas implicações.

Nos encontros, atentou-se também à comunicação não verbal das mulheres, suas expressões, seus olhares, o sorriso ou o choro, com o objetivo de compreender o significado daquela experiência para cada uma, pois na prática educativa é importante saber escutar os envolvidos e reconhecer suas necessidades. "Escutar significa a disponibilidade permanente 
por parte do sujeito que escuta para a abertura à fala do outro, ao gesto do outro e às diferenças do outro"18.

Outra questão observada é o potencial de um grupo para incitar a transformação de saberes e práticas, que é evidenciado pela presença de fatores terapêuticos, como oferecimento de informações, coesão do grupo, desenvolvimento de técnicas de socialização, aprendizagem interpessoal, entre outros ${ }^{19}$. As mulheres participantes deste estudo já sinalizaram essa transformação, mediante tudo o que foi evidenciado durante os encontros com o grupo, por meio de seus comentários na aprendizagem sobre a discussão dos temas em saúde.

$\mathrm{Na}$ educação problematizadora, não ocorre, na maioria das vezes, a transformação imediata da realidade, que só é percebida quando o sujeito "der marcas de transformação do seu saber", que acontece em processos contínuos, na intermediação dos saberes científicos e populares para a conquista de um novo saber ${ }^{20}$.

Os elementos sinalizados nesse processo educativo em saúde, como autonomia, cidadania e consciência crítica, são aspectos que devem estar presentes em processos educativos que assegurem a participação das pessoas.

As participantes acreditam que podem compartilhar o conhecimento adquirido com outras mulheres da comunidade e com sua família. Esse fato é positivo, pois o processo educativo se inicia na família, que é uma instituição na qual os sujeitos iniciam sua formação, constituindo-se como a base da incorporação de padrões de comportamento, valores morais, sociais, éticos e espirituais, entre outros ${ }^{21}$ e sendo as mulheres participantes, em sua maioria, cuidadoras da família, os saberes formados no grupo contribuirão significativamente para a incorporação de novos saberes e práticas.

\section{CONSIDERAÇÕES FINAIS}

Nas atividades de Educação em Saúde, a participação dos sujeitos deve ser o elemento norteador, pois, quando estão envolvidos, sentem-se mais motivados e, consequentemente, corresponsáveis pela sua saúde e pela mudança da realidade.

A estratégia educativa desenvolvida nos encontros com o grupo de mulheres é contrária a programações pré-estabelecidas pelos profissionais de saúde, muitas vezes rígidas, excludentes e ineficientes.

A experiência desenvolvida certamente incrementou o arsenal de conhecimento dessas mulheres sobre sua saúde, contribuindo, assim, para a promoção da saúde e a autoestima.

A partir deste estudo, que descreveu atividades educativas desenvolvidas pela ESF com mulheres adultas jovens na comunidade, espera-se contribuir no campo de atuação na ESF, especialmente para a prática educativa em saúde, demonstrando que é possível desenvolver atividades educativas com a comunidade, de acordo com cada realidade e necessidade, pautadas no diálogo e na participação dos sujeitos.

Incentivam-se os profissionais de saúde a desenvolverem atividades educativas diferenciadas, com foco nas necessidades locais, respeitando a cultura e o contexto e em parceria com a comunidade, contribuindo, assim, para a promoção da saúde.

Sugere-se a realização de estudos posteriores, utilizando métodos participativos, que tragam novamente à tona questões como essas, para que, cada vez mais, a temática de Educação em Saúde faça parte das rodas de discussão entre os profissionais de saúde, com o intuito de aprimoramento dessa prática.

\section{REFERÊNCIAS}

1. Campos RTO, Campos GWS. Co-construção de Autonomia: o sujeito em questão. In: Campos GWS, Minayo MCS, Akerman M, Drumond Junior M, Carvalho YM. (org.) Tratado de Saúde Coletiva. São Paulo: Hucitec-Fiocruz; 2006. p. 669-87.

2. Cervera DPP, Parreira BDM, Goulart BF. Educação em saúde: percepção dos enfermeiros da atenção básica em Uberaba (MG). Ciênc Saúde Coletiva. 2011;16(1):1547-54

3. Pedrosa JIS. Educação popular no ministério da saúde: identificando espaços e referências. In: Ministério da Saúde. Secretaria de Gestão Estratégica e Participativa. Departamento de Apoio à Gestão Participativa. Caderno de Educação Popular e Saúde. Brasília: Ministério da Saúde; 2007.

4. Ministério da Saúde. Saúde da Família: Uma Estratégia para a reorientação do modelo assistencial. Brasília: Ministério da Saúde; 1997.

5. Acioli SA. A prática educativa como expressão do cuidado em saúde pública. Rev Bras Enferm. 2008;61:117-21.
6. Roecker S, Marcon SS. Educação em saúde na estratégia saúde da família: o significado e a práxis dos enfermeiros. Esc Anna Nery Rev Enferm. 2011;15(4):701-9.

7. Sousa LB, Torres CA, Pinheiro PNC, Pinheiro AKB. Práticas de educação em saúde no Brasil: a atuação da enfermagem. Rev Enferm UERJ. 2010;18:55-60.

8. Sousa LB, Aquino OS, Fernandes JFP, Vieira NFC, Barroso MGT. Educação, cultura e participação popular: Abordagem no contexto da educação em saúde. Rev Enferm UERJ. 2008;16(1):107-12.

9. Ministério da Saúde. Secretaria de Atenção à Saúde. Departamento de Ações Programáticas Estratégicas. Política nacional de atenção integral à saúde da mulher: princípios e diretrizes. Brasília: Ministério da Saúde; 2004.

10. Wall ML. Tecnologias educativas: subsídios para a assistência de enfermagem em Grupos. Goiânia (GO): AB; 2001.

11. Ministério da Saúde. Conselho Nacional de Saúde. Resolução nº 196 de 10 de outubro de 1996. Pesquisa envolvendo seres humanos. Bioética. 1996;4:15-25. 
12. Acker JIBV, Cartana MHF.Construção da participação comunitária para a prevenção de acidentes domésticos infantis. Rev Bras Enferm. 2009;62(1):64-70.

13. Trindade WR, Ferreira MA. Grupo feminino de cuidado: estratégia de pesquisa-cuidado à mulher. Rev Bras Enferm. 2009;62(3):374-80.

14. Santos RV, Penna CMM. A educação em saúde como estratégia para o cuidado à gestante, puérpera e ao recém-nascido. Texto \& Contexto Enferm. 2009;18(4): 652-60.

15. Alves GG, Aerts D. As práticas educativas em saúde e a Estratégia Saúde da Família. Ciênc Saúde Coletiva. 2011;16(1):319-25.

16. Hoga LAK, Reberte LM. Pesquisa-ação como estratégia para desenvolver grupo de gestantes: a percepção dos participantes. Rev Esc Enferm USP. 2007;41:559-66.

17. Leonello VM, Oliveira MAC. Construindo o diálogo entre saberes para ressignificar a ação educativa em saúde. Acta Paul Enferm. 2009;22(Especial - 70 Anos):916-20.
18. Freire P. Pedagogia da autonomia: saberes necessários à prática educativa. São Paulo: Paz e Terra; 1996; 148 p.

19. Oliveira NF, Munari DB, Bachion MM, Santos WS, Santos QR. Fatores terapêuticos em grupos de diabéticos. Rev Esc Enferm USP. 2009;43:558-65.

20. Alvin NAT, Ferreira MA. Perspectiva problematizadora da Educação Popular em Saúde e a Enfermagem. Texto \& Contexto Enferm. 2007;16(2):315-9.

21. Macedo VCD, Monteiro ARM. Educação e saúde mental na família: experiência com grupos vivenciais. Texto \& Contexto Enferm. 2006;15(2):222-30.

Recebido em: 29/09/2012

Aprovado em: 12/08/2013 